\title{
Descolonizar e interculturalizar el saber: el reto de la universidad latinoamericana.
}

\author{
Galarza López, C. Gabriel \\ Consejo de Aseguramiento de la Calidad de \\ la Educación Superior CACES, Ecuador \\ gabriel.galarza@caces.gob.ec
}

\author{
Salazar, Alba \\ Universidad Estatal de Bolívar, Ecuador \\ albasalazarguerrero@gmail.com
}

\section{Resumen}

Varios intelectuales latinoamericanos, en los últimos años, vienen reflexionando acerca de lo que ha significado y lo que aún significa en la actualidad, para América Latina y el Caribe la modernidad generada en Occidente y la cara oculta de la colonialidad. Entre ellos, consideramos que Aníbal Quijano constituye un referente que vigoriza el pensamiento crítico latinoamericano, a partir de la teoría de la colonialidad del poder. Mediante la deconstrucción histórica devela la existencia de un patrón de poder de dominación social que se engendra en el siglo XVI con la conquista y colonización, cuyas ideas se han prolongado hasta nuestros días. La idea de raza es un constructo mental que viene operando eficazmente como mecanismo de subalternidad y exclusión de grupos humanos, así como el patriarcado, la hegemonía cultural y el uso indiscriminado de la naturaleza para sobreexplotarla y degradarla. Todo ello para saciar la voracidad del capitalismo.

El saber occidental se constituyó en una estructura de dominación y hegemonía del poder colonial. Los conocimientos eurocéntricos se constituyeron en universales y se transmiten mediante cánones de al- ta valoración, de acuerdo con sus intereses económicos y políticos. Como contraparte degrada y descalifica a conocimientos emergidos desde la diversidad cultural de pueblos originarios.

Palabras clave: Modernidad / Colonialidad del poder; del saber y del ser; decolonialidad; subalternidad; exclusión sociocultural; diversidad cultural; interculturalidad;

\section{Abstract}

Several Latin American intellectuals, in recent years, have been reflecting on what it has meant and what it still means at present, for Latin America and the Caribbean, the modernity generated in the West and the hidden side of coloniality. Among them, we consider that Aníbal Quijano constitutes a reference that invigorates Latin American critical thinking, based on the theory of the coloniality of power. Through historical deconstruction reveals the existence of a pattern of power of social domination that is engendered in the sixteenth century with the conquest and colonization, whose ideas have continued to this day. The idea of race is a mental construct that has been operating effectively as a mechanism of su- 
balternity and exclusion of human groups, as well as patriarchy, cultural hegemony and the indiscriminate use of nature to overexploit and degrade it. All this to satisfy the voracity of capitalism.

Western knowledge was constituted in a structure of domination and hegemony of colonial power. Eurocentric knowledge became universal and is transmitted through high valuation canons, according to its economic and political interests. As a counterpart, it degrades and disqualifies knowledge emerging from the cultural diversity of indigenous peoples.

Keywords: Modernity / Coloniality of power; of knowledge and being; decoloniality; subalternity; sociocultural exclusion; cultural diversity; interculturality;

\section{INTRODUCCIÓN}

"El cambio de mentalidad es una transformación profunda" ( $L$. Proaño)

La colonialidad para América Latica y el Caribe, no solo debe ser abordada como un hecho histórico, sino como un fenómeno actual y omnipresente que subyuga a nuestros pueblos. La neocolonialidad representa un orden social jerárquico de poder, establecido desde la hegemonía del capital financiero que demanda de seres humanos racializados, explotados e inferiorizados, involucra lo económico, polí- tico, religioso, educativo, cultural, militar, ecológico; además de aqueIlos universos subjetivos que trastocan desde la existencia misma de los seres humanos, sus conocimientos, su filosofía, lo psicológico y cuyo denominador común es el dominio de unos seres humanos sobre otros.

"La colonialidad representa una gran variedad de fenómenos que abarcan una serie de fenómenos desde lo psicológico y existencial, pasando por lo cultural y educativo, hasta lo económico y militar, y que tienen una característica común: la determinación y dominación de uno por otro, de una cultura, cosmovisión, filosofía, religiosidad y un modo de vivir por otros del mismo tipo" (Estermann, 2015. 115).

Ligado a lo dicho, debe considerarse que los estudios de procesos de la inteculturalidad son posibles descifrarlos, explicarlos e interpretarlos desde una postura de relaciones de poder, ello nos permite comprender por qué es imprescindible establecer correlatos entre la praxis del concepto y la colonialidad del poder, del saber y del ser. Esta línea de pensamiento nos permite trasladar las reflexiones epistémicas del ámbito educativo, 
al ámbito social para ubicarlo en referencia a las estructuras de dominación. En esto coincidimos con Walsh cuando dice que, "La interculturalidad sin la decolonialidad no tiene el mismo sentido crítico y transformador" (Walsh, 2012. 18).

Para acercarnos al tema nos apoyamos en valiosos criterios de intelectuales latinoamericanos/as, entre ellos/as tomamos como referencia la propuesta de la teoría de la colonialidad del poder del sociólogo peruano Aníbal Quijano, además de diversos aportes que han realizado otros/as pensadores de diferentes latitudes del mundo quienes han advertido las inequidades del poder hegemónico de la cultura occidental eurocéntrica. Con sus propuestas y desde una teoría critica han irrumpido en el quehacer de las ciencias sociales y humanas normalizadas, donde se advierte una incesante búsqueda de alternativas para subvertir patrones de dominación de este modelo de desarrollo del sistema capitalista, racista y patriarcal.

Estos elementos marcan la dinámica del sistema moderno - capitalista $y$ de todas sus formas de explotación económica, social, cultural y de la naturaleza con fines acumulativos. Es a partir de estos aportes que Quijano demuestra una serie de elementos coloniales que subsisten arraigados como parte de nuestra herencia de cultura colonizada.

Patrones desarrollados por A. Quijano, se subrayan los siguientes enunciados de gran valor instrumental para los análisis sociológicos de la interculturalidad, ellos son:

- La idea de raza fue un modo de otorgar legitimidad a las relaciones de dominación impuestas por la conquista. Esta aún es reproducida en las prácticas sociales al estar fuertemente estructurada en relaciones.

- Todas las formas de control y de explotación del trabajo y de control de la producción-apropiación-distribución de productos, continúan siendo articuladas alrededor de la relación capital-salario-mercado mundial. Subsisten la servidumbre, nuevas formas de esclavitud, la pequeña producción mercantil, la reciprocidad y el salario.

- El aprendizaje forzoso de la cultura impuesta por los colonizadores/dominadores ha 
condicionado a largo plazo una colonización de las perspectivas cognitivas, de los modos de producir u otorgar sentido a los resultados de la experiencia material o intersubjetiva, del imaginario, del universo de relaciones intersubjetivas del mundo, que persiste hasta hoy.

La trilogía colonialidad del poder, del saber y del ser, en los últimos años ha favorecido los análisis sociológicos de la interculturalidad, impactando en los espacios políticos, educacionales, gubernamentales, jurídicos y comunitarios.

\section{LA COLONIALIDAD DEL PODER}

Es un patrón de dominación global propio del sistema capitalista, originado con la conquista y el colonialismo europeo en el siglo XVI, el cual establece su hegemonía a partir de dos elementos constituyentes. Por una parte, la clasificación de la población mundial sobre la idea de raza, y por el otro, el control del trabajo y la articulación de todos sus recursos y productos en torno al capital y al mercado mundial (Quijano 2000: 202).
Es preciso aclarar que la idea de raza es un constructo sociocultural, siendo una inventiva de los colonizadores, para justificar el dominio de unos seres humanos sobre otros, a quienes incluso denominaron "naturales" desde una perspectiva de mirar al otro como inferior.

La superioridad racial se estableció mediante una clasificación taxonómica de los humanos (Castro-Gómez, 2000: 153). Esta superioridad se afirmó a través de una supuesta estructura biológica, auto-otorgada a la proclamada raza "blanca". Elementos que supusieron les hacían superiores y provistos de razón, con aptitudes para la ciencia y la técnica, mientras el resto de la población por su color de piel eran inferiores y desprovistos de razón, aptos únicamente para los oficios subalternos y manuales. Así se fue creando el patrón cultural de superioridad - inferioridad. (Noboa, 2005:76), (Gómez, 2017. 107).

La clasificación racial y la división del trabajo no solo debe ser tratada y vista como un hecho histórico colonial, sino como un fenómeno constitutivo desde la sub- 
jetividad social moderna, como una construcción cultural hegemónica que por más de cinco siglos secuestra nuestras conciencias, y los valores humanos.

La colonización forjó nuevas identidades en América de acuerdo a las características étnico culturales, especialmente fenotípicas, las que fueron valoradas desde la visión hegemónica y etnocéntrica sin distinción, (aymaras, incas, mayas, aztecas, mapuches, etc.). Todos los habitantes de las culturas americanas eran cobijados por el término segregador de indios; de esta manera, la rica diversidad cultural de los pueblos originarios quedó homogenizada y subalternizada a la servidumbre (Kowii, 2011: 16).

Pero, además, para la explotación del trabajo, extracción de los recursos mineros y tareas minimizadas se introdujeron ingentes cantidades de esclavizados africanos, quienes fueron comercializados como simples mercancías. De todos los grupos humanos, los africanos fueron los más explotados y reduci- dos a la esclavitud, pues su trabajo gratuito representaba la parte principal de la economía capitalista naciente por ser más resistentes para realizar los trabajos en climas tropicales duros, especialmente en los yacimientos de oro, piedras preciosas, y la agricultura ${ }^{1}$.

El control del trabajo y la imposición de patrones etno-culturales determinaron la distribución geográfica de cada una de las formaciones sociales de producción para el mercado mundial, lo cual confinó a América a un rango inferior de periferia, con culturas subalternas y encargada de la producción de materias primas, incluso productos agrícolas originarios que fueron utilizados en beneficio de las poblaciones del norte, mientras tanto Europa se fue consolidando como el centro del mundo y el desarrollo capitalista en sus diversas fases, gracias, y en gran parte, a la acumulación de capital proveniente de las riquezas del nuevo mundo y el trabajo subordinado de los colonizados.

1. Los indios fueron confinados a la servidumbre ya que su contextura física no era tan resistente como los africanos. Entre ellos, hubo una clase india, los caciques, que tenía ciertos privilegios por ser los intermediarios entre blancos e indios. Los "blancos" amparados en la superioridad otorgada por la distribución racial, eran los únicos que tenían derecho a recibir salario por el trabajo que desempeñaban. 


\section{LA COLONIALIDAD DEL SABER}

El saber es otro de los elementos constitutivos de la colonialidad del poder eurocéntrico, se define como un universo de relaciones subjetivas de dominación, lo cual configura un nuevo escenario de relaciones geo-culturales (Quijano, 2000.209). Los colonizadores además de imponer sus conocimientos se apropiaron de los conocimientos de los colonizados, aquellos que eran útiles como medio de explotación social y de la naturaleza, para consolidar el capitalismo como modelo único en el mundo.

"El logocentrismo es la sintesis histórica del saber y del poder, sustentada en la unidad entre totalidad y verdad histórica. La colonialidad opera precisamente para desacralizar esta unidad y mostrar que esta relación está inserta en condiciones históricas completas; es decir, no hay una historia de la episteme que responda a su propia lógica interna, sino una episteme relacional y respectiva; o intrinsecamente emparentada con la doxa y con los procesos históricos. Así, por ejemplo, la incorporación de América a la clasificación geográfica e histórica, responde a un momento gnoseológico marcado por los intereses del poder $y$ del saber colonial" (León. 2005: 121).

Para Castro-Gómez el conocimiento actúa desde la subjetividad para someter a los seres humanos a seguir un patrón epistemológico único, establecido y normado por la modernidad. "Someter la vida entera al control absoluto del hombre bajo la guía segura del conocimiento" (Castro-Gómez, 2000:146).

En el siglo XIX al separarse de las matemáticas, astronomía y biología, se desarrollan y se consolidan las ciencias sociales como expresión eurocéntrica de enunciación del conocimiento, como verdad única del "saber y del decir, del conocer y su expresión" (Garcés, 2005: 142). La valoración e imposición epistemológica trazó hasta hoy una ruta del poder que valora y delimita qué saber debe ser considerado como tal, en que idiomas o lenguas deben ser enunciados, frente a otros saberes y lenguas que quedan silenciados en la matriz colonial. 
Las ciencias sociales al ser pensadas y construidas estructuralmente desde el patrón del poder moderno/colonial, para algunos, se han convertido en el aparato de soporte ideológico del sistema. Por una parte - hacia dentro del Estadonación moderno actúa en la subjetivad sociocultural, lo cual garantiza mantener el control y el orden del aparato productivo capitalista; por otra parte -hacia fuera- legitima la división del trabajo y el intercambio desigual.

Así las ciencias sociales (antropología, psicología, historia, sociología, etnografía etc.) han venido mediando y resguardando los grandes beneficios económicos y sociales que obtienen los países del centro al costo del dominio y exclusión de las periferias.

"Nuestra tesis es que las ciencias sociales se construyen en este espacio de poder moderno/colonial y en los saberes ideológicos generados por él. Desde este punto de vista las ciencias sociales no efectuaron jamás una ruptura epistemológica" (Castro-Gómez, 2000:153).

\section{LA COLONIALIDAD DEL SER}

El dominio militar se impuso con extrema violencia y sadismo, eran verdaderos carniceros de humanos sin distinción de género ni edad. El fenómeno militar consiste en el sometimiento de los cuerpos de los/as indios/as mediante la fuerza extrema para la explotación laboral no remunerada, muchas veces hasta la muerte.

"Esos pueblos fueron conquistados por invasores europeos desde el siglo XVI. Se les impuso leyes, costumbres y religión; se les sometió a la explotación y a la muerte" (Ayala, 2011. 37).

El poder militar además actúa de forma morbosa sobre los cuerpos de las indias que eran utilizadas como objetos sexuales para saciar los más bajos instintos de los colonizadores. Por otra parte, trastoca las sensibilidades más íntimas de los subalternizados ya que involucra todo el universo subjetivo, la psiquis, los sentimientos, es la dignidad humana degradada a la impotencia y miseria. "La "colonización" o el dominio del cuerpo de la mujer india es parte de una cultura que se basa también en el dominio del 
cuerpo del varón indio. A este se lo explotará principalmente por el trabajo" (Dussel, 2008. 50). La tiranía del verdugo no solo era amolar los cuerpos hasta la muerte sino herir en lo más profundo de la dignidad del indio al ver explotadas laboral y sexualmente a las mujeres de su círculo familiar y comunitario (esposa, hijas, hermanas), reducidas a la servidumbre y como esclavas sexuales.

"En 1492, los nativos descubrieron que eran indios, descubrieron que vivian en América, descubrieron que estaban desnudos, descubrieron que existía el pecado, descubrieron que debian obediencia a un rey y a una reina de otro mundo y a un dios de otro cielo, y que ese dios habia inventado la culpa y lo vestido y habia mandado que fuera quemado vivo quien adorara al sol y a la luna y a la tierra y a la lluvia que moja”. (Galeano, 2012: 7)

La colonialidad del ser es un fenómeno militar, cultural, de la naturaleza, entendida como parte del ser humano donde se desarrolla la vida misma, es el dominio de los cuerpos, de la sexualidad, las subjetividades, las sensibilidades y los imaginarios (Guerrero, 2011.74). Para mantener el control de la cla- se social en condición de subalternidad reprimieron cuanto fue posible todo su universo simbólico, sus sentidos, sus costumbres, sus expresiones, sus sentimientos, sus valores. Los indios fueron violentados en lo más profundo y sensible del ser humano, despojándolos de gran parte de su herencia y vivencia cultural, de conocimientos y cosmovisiones que es la identidad misma.

\section{DESCOLONIZAR E}

\section{INTERCULTURALIZAR EL SABER}

Desde la teoría de la colonialidad del poder, concepción introducida, sobre todo por A. Quijano, la sociología latinoamericana ha comenzado a vislumbrar una nueva ruta epistemológica, la que permite explicar los actuales procesos en los que se debate la praxis de las relaciones de poder, subalternidad y exclusión socio-cultural, que encuentra ecos en la interculturalidad.

Descolonizar e interculturalizar el saber significa un gran reto para la universidad latinoamericana, no solo por el hecho de interiorizar y aceptar su condición de coloniali- 
dad sino por el papel que históricamente ha cumplido como gestora, muchas veces inconsciente, de la perpetuidad del poder colonizador.

El desafío para la universidad es comenzar a escribir, desde otros horizontes epistemológicos, nuestra propia historia, a partir del desmontaje de estructuras objetivas y subjetivas impuestas, pasando por las políticas, objetivos, estrategias, la producción y validación de conocimientos, los currículos, las valoraciones de ingreso y graduación, los métodos de aprendizaje y enseñanza y sobre todo las relaciones verticales de los actores sociales. Caso contrario la educación universitaria no podrá ser descolonizadora.

El traspaso de una universidad anacrónica hacia una institución descolonizada, descolonizadora e intercultural no es nada fácil, requiere de la reflexión profunda de los protagonistas, es devolverle su legitimidad social, transformarse en un ente activo del desarrollo del contexto, el replanteamiento epistémico que parta desde la recuperación de los conocimientos ancestrales, la producción de cono- cimientos propios, la reflexión, validación y aceptación ética de conocimientos científicos y tecnológicos que sean útiles para el desarrollo social, el diálogo de saberes, y el respeto y conservación de la naturaleza.

Las universidades están convocadas a cambiar la historia socio - cultural de Latinoamérica. La educación superior hasta la actualidad, salvo pocas excepciones, ha sido útil para que se perennice la colonialidad en todas sus expresiones. Debe ser el instrumento de liberación de nuestros pueblos, de la construcción de la autonomía de sus profesionales y académicos, transgredir a conceptos y conocimientos que impidan del involucramiento con la sociedad y la relación dialógica con las diversas culturas, con el uso de tecnologías apropiadas y diversas, que sean el producto de la investigación y la innovación científica y tecnológica, el diálogo de saberes que, favorezcan a la resolución de los problemas sociales, a la praxis intercultural, y aporten a la sociedad hacia las grandes conquistas de la humanidad. 
En nuestro caso ecuatoriano, en la Constitución del año 2008, el país es declarado como un Estado intercultural y plurinacional, por una parte. Dicha declaratoria no ha sido suficiente para que la interculturalidad llegue a ser una praxis institucional y social, por el contrario, la interculturalidad se encuentra atrapada en el concepto y en la norma.

Por otra parte, al convertirse en discurso únicamente estatal y no afincado en la sociedad, ha perdido paulatinamente su poder de convocatoria y movilización, sobre todo, incluso por parte de las nacionalidades, pueblos indígenas y afrodescendientes, quienes las plantearon entre sus grandes reivindicaciones, desde aproximadamente los años sesenta del siglo pasado.

No obstante, es necesario destacar que unas pocas universidades en el país generaron carreras de grado y posgrado, especialmente tratando el tema de la interculturalidad, tales como la Universidad Estatal de Bolívar, la Universidad Andina Simón Bolívar, la Universidad Politécnica Salesiana entre otras. Hay otras que incluyeron su tratamiento de los procesos de for- mación en diversas carreras como derecho, enfermería, sociología, antropología etc.

El reglamento de régimen académico aprobado por el Consejo de Educación Superior (CES), de igual manera exigió su tratamiento, y finalmente fue creada la universidad de tinte particular Amawta Wasi, pero que dejó de funcionar, siendo en la actualidad una universidad declarada pública y comunitaria, la que debe iniciar su funcionamiento en los próximos meses, de acuerdo a la reforma de la Ley Orgánica de Educación Superior, publicada el 2 de agosto del 2018.

Es indiscutible las luchas que han llevado a cabo desde la época colonial los pueblos indígenas y afrodescendiantes, exigiendo sus derechos, los que han sido tratados en la época republicana, generando organizaciones como la Federación Ecuatoriana de Indios (FEI) en el año 1944, más tarde el Movimiento Ecuarunari y la CONAIE etc. $E$ inclusive grandes levantamientos nacionales que obligaron al Estado a llevar el reconocimiento de la diversidad étnica y cultural en las Constituciones de 1998 y 2008. 
Empero las lacras subsistentes en el espíritu eurocentrista afincado en América Latina, y específicamente en el Ecuador, aún no logran ser comprendidas en muchos sectores ciudadanos. Esto se observa sobre todo en los territorios que aún no se han interpuesto profundamente políticas reales de transformación de las estructuras neocoloniales de raigambre histórica de la sobreviviente colonialidad.

Las universidades y las demás instituciones de educación superior, así como el esfuerzo del sistema educativo nacional, con diversos ritmos de avance territorial están imprimiendo políticas decoloniales e interculturales. Una de ellas, en el sistema de educación superior es la articulación de las funciones sustantivas de la docencia con la vinculación con la sociedad, la investigación e innovación sobre todo de carácter social.

El trabajo con los sectores comunitarios indígenas, afrodescendientes y montubios, los migrantes a los barrios periféricos de las ciudades, permitirán a los profesores, investigadores y estudiantes, no sólo conocer sus realidades humanas, sino interpretarlas, y despertar la sensibilidad humana, coanalizar sus problemas, buscar conjuntamente soluciones, abrir la conciencia, trasladar saberes y situaciones al aula, investigar y proponer innovaciones dialogadas y trabajadas con los grupos humanos etc.

\section{CONCLUSIÓN}

Finalmente, reiteramos que la realidad de la vida humana es compleja. $Y$ puede llevar a los universitarios a tratarla de manera multi e interdisciplinaria, lo que es loable llevarlo a la práctica. $Y$, en la búsqueda de soluciones, particularmente al abordar, realizar, participar en proyectos de desarrollo local, tener en cuenta que estos deben ser entendidos desde el dialogo permanente, sabiendo de antemano que los sectores indígenas y afrodescendientes poseen muchos conocimientos, no necesariamente calificados como científicos, pero que aportan y han aportado interpretaciones y soluciones pertinentes para el desenvolvimiento de sus vidas. Articular tales saberes a los conocimientos científicos y tecnológicos serán de una inquebrantable riqueza de las posibilidades humanas de transformación. 
La interdisciplina pretende comprender la realidad desde sus variadas dimensiones, ya sean económicas, políticas, culturales, sociales y ambientales. Y sobre todo en nuestra América la transformación de las estructuras económico políticas y culturales, en la educación debe tocar necesariamente al cambio de viejas barreras, asentadas en las estructuras mentales y en las actitudes humanas.

Para Boaventura de Sousa Santos la teoría decolonial atraviesa por la dificultad de superar el colonialismo no solo como política del Estado, sino que debe ser superada desde los constructos mentales y culturales, enraizados en todas las estructuras sociales. "es una gramática social muy vasta que atraviesa la sociabilidad, el espacio público y el espacio privado, la cultura, las mentalidades y las subjetividades". (Sousa - Santos, 2010. 15).

\section{BIBLIOGRAFÍA}

Ayala, Enrique (2011). La interculturalidad: camino para el Ecuador. En: Interculturalidad y diversidad. Quito Ecuador: Corporación Editora Nacional
Castro-Gómez, Santiago (2000). Ciencias sociales, violencia epistémica y el problema de la "invención del otro". En: Edgardo Lander. La colonialidad del saber: eurocentrismo y ciencias sociales perspectivas latinoamericanas. Buenos Aires - Argentina: CLACSO.

Constitución. (2008). Asamblea Nacional del Ecuador. Quito, Ecuador.

Dussel, Enrique (2008). 1492 el encubrimiento del otro. Hacia el origen del "mito de la modernidad". La Paz - Bolivia: Biblioteca Indígena.

Estemann, Josef (2015). Más allá de occidente. Apuntes filosóficos sobre interculturalidad, descolonización y el Vivir Bien andino. Quito - Ecuador: Abya-Yala

Galeano, Eduardo (2012). Los hijos de los días. En: Indios, negros y otros indeseables. Capitalismo, racismo y exclusión en América Latina y el Caribe. Quito: Abya-Yala.

Garcés, Fernando (2005). Las políticas del conocimiento y la colonialidad lingüística y epistémica. Quito - Ecuador: Abya-Yala.

Gómez, Jorge (2017). Aproximaciones semióticas a la interculturalidad. Guayaquil - Ecuador: UArtes Ediciones.

Guerrero, Patricio (2011). Interculturalidad y plurinacionalidad, escenarios de lucha de sentidos: entre la usurpación y la insurgencia simbólica. En: Interculturalidad y diversidad. Quito - Ecuador: Corporación Editora Nacional.

Kowii, Ariruna (2011). Diversidad e interculturalidad. En: Interculturalidad y diversidad. Quito - Ecuador: Corporación Editora Nacional. 
León, Catalina (2005). Hacia una posible superación de la metahistoria de lo latinoamericano. Quito - Ecuador: Abya-Yala.

Noboa, Patricio (2005). La matriz colonial, los movimientos sociales y los silencios de la modernidad. Quito - Ecuador: Abya-Yala.

Quijano, A. (2000). Colonialidad de poder, eurocentrismo y América Latina, Lima, Perú

Sousa, Boaventura (2010). Descolonizar el saber, reinventar el poder. Uruguay: Don Bosco.

Walsh, Katherine (2012). Interculturalidad crítica y (de)colonialidad. Ensayos desde Abya-Yala. Quito - Ecuador: AbyaYala 